\title{
Adaptive solution of a singularly-perturbed convection-diffusion problem using a stabilized mixed finite element method
}

\author{
María González ${ }^{1}$ and Magdalena Strugaru ${ }^{2}$ \\ 1 Universidade da Coruña, Campus de Elviña s/n, 15071, A Coruña, Spain, \\ maria.gonzalez.taboada@udc.es \\ 2 Basque Center of Applied Mathematics, Alameda Mazarredo 14, 48009, Bilbao, \\ Spain, mstrugaru@bcamath.org
}

\begin{abstract}
We explore the applicability of a new adaptive stabilized dual-mixed finite element method to a singularly-perturbed convection-diffusion equation with mixed boundary conditions. We establish the rate of convergence when the flux and the concentration are approximated, respectively, by Raviart-Thomas/BrezziDouglas-Marini and continuous piecewise polynomials. We consider a simple a posteriori error indicator and provide some numerical experiments that illustrate the performance of the method.
\end{abstract}

\section{Introduction}

The numerical solution of convection-dominated convection-diffusion problems is difficult. We are interested in the simultaneous approximation of the concentration and the flux in a singularly-perturbed convection-diffusion equation with mixed boundary conditions. This type of mixed methods was first proposed and analyzed by J.M. Thomas [12].

The use of augmented mixed finite element methods allows to use a wider set of finite element subspaces in the discretization (see $[9,5,1,6,2]$ and the references therein). However, in $[6,2]$ only homogeneous boundary conditions of Dirichlet type were treated.

In this work we analyze the applicability of adaptive augmented mixed finite element methods to a singularly-perturbed convection-diffusion equation with mixed boundary conditions. In Section 2 we describe the augmented dual-mixed variational formulation. In Section 3 we present the stabilized mixed finite element method. In Section 4, we introduce a new a posteriori error indicator that is reliable and locally efficient. Finally, numerical experiments are reported in Section 5.

\section{Augmented dual-mixed variational formulation}

Let $\Omega$ be a bounded domain of $\mathbb{R}^{2}$ with a Lipschitz-continuous boundary $\Gamma$. We assume that $\Gamma$ is decomposed into two disjoint open parts, $\Gamma_{D}$ and $\Gamma_{N}$. Let 
$\mathbf{b} \in\left[L^{\infty}(\Omega)\right]^{2}$ be solenoidal in $\Omega$. Then, given $f \in L^{2}(\Omega), g \in H^{1 / 2}\left(\Gamma_{D}\right)$ and $z \in H^{-1 / 2}\left(\Gamma_{N}\right)$, we consider the problem: find $\sigma: \Omega \rightarrow \mathbb{R}^{2}$ and $u: \Omega \rightarrow \mathbb{R}$ such that

$$
\left\{\begin{aligned}
-\operatorname{div}(\sigma)+\mathbf{b} \cdot \nabla u=f & \text { in } \Omega, \\
\epsilon^{-1} \sigma-\nabla u=0 & \text { in } \Omega, \\
u=g & \text { on } \Gamma_{D}, \\
\sigma \cdot \mathbf{n}=z & \text { on } \Gamma_{N},
\end{aligned}\right.
$$

where $\epsilon>0$ is a parameter and $\mathbf{n}$ is the unit outward normal vector to $\Gamma$.

Given $s \in H^{-1 / 2}\left(\Gamma_{N}\right)$, we define the space $H_{s}:=\{\tau \in H(\operatorname{div} ; \Omega): \tau \cdot \mathbf{n}=$ $s$ on $\left.\Gamma_{N}\right\}$ and decompose $\sigma$ as $\sigma=\sigma_{0}+\sigma_{z} \in H_{0}+H_{z}$. We consider the bilinear forms $a: H_{0} \times H_{0} \rightarrow \mathbb{R}, b: H^{1}(\Omega) \times H_{0} \rightarrow \mathbb{R}$ and $c: H^{1}(\Omega) \times$ $H^{1}(\Omega) \rightarrow \mathbb{R}$ defined by

$$
a(\zeta, \tau):=\frac{1}{\epsilon} \int_{\Omega} \zeta \cdot \tau, \quad b(w, \tau):=\int_{\Omega} w \operatorname{div}(\tau), \quad c(w, v):=\int_{\Omega} \mathbf{b} \cdot \nabla w v
$$

for all $\zeta, \tau \in H_{0}$ and $w, v \in H^{1}(\Omega)$, and the linear functionals $m: H_{0} \rightarrow \mathbb{R}$ and $l: H^{1}(\Omega) \rightarrow \mathbb{R}$ defined by

$$
m(\tau):=\int_{\Gamma_{D}} g \tau \cdot \mathbf{n}-\frac{1}{\epsilon} \int_{\Omega} \sigma_{z} \cdot \tau, \quad l(v):=-\int_{\Omega}\left(f+\operatorname{div}\left(\sigma_{z}\right)\right) v,
$$

for all $\tau \in H_{0}$ and $v \in H^{1}(\Omega)$.

We then consider the following augmented variational formulation of problem (1): find $\left(\sigma_{0}, u\right) \in \mathbf{H}:=H_{0} \times H^{1}(\Omega)$ such that

$$
A_{s}\left(\left(\sigma_{0}, u\right),(\tau, v)\right)=F_{s}(\tau, v), \quad \forall(\tau, v) \in \mathbf{H},
$$

where $\mathbf{H}$ is endowed with the product norm and the bilinear form $A_{s}: \mathbf{H} \times$ $\mathbf{H} \rightarrow \mathbb{R}$ and the linear functional $F_{s}: \mathbf{H} \rightarrow \mathbb{R}$ are defined by

$$
\begin{gathered}
A_{s}((\zeta, w),(\tau, v)):=a(\zeta, \tau)+b(w, \tau)-b(v, \zeta)+c(w, v) \\
\quad+\kappa_{1} \int_{\Omega}(\operatorname{div}(\zeta)-\mathbf{b} \cdot \nabla w)(\operatorname{div}(\tau)+\mathbf{b} \cdot \nabla v) \\
+\kappa_{2} \int_{\Omega}\left(\nabla w-\epsilon^{-1} \zeta\right) \cdot\left(\nabla v+\epsilon^{-1} \tau\right)+\kappa_{3} \int_{\Gamma_{D}} w v
\end{gathered}
$$

and

$$
\begin{aligned}
F_{s}(\tau, v) & :=m(\tau)-l(v)-\kappa_{1} \int_{\Omega}\left(f+\operatorname{div}\left(\sigma_{z}\right)\right)(\operatorname{div}(\tau)+\mathbf{b} \cdot \nabla v) \\
& +\frac{\kappa_{2}}{\epsilon} \int_{\Omega} \sigma_{z}\left(\nabla v+\epsilon^{-1} \tau\right)+\kappa_{3} \int_{\Gamma_{D}} g v
\end{aligned}
$$

for all $(\zeta, w),(\tau, v) \in \mathbf{H}$.

We have the following result concerning the well-posedness of problem (4). 
Theorem 1. Assume that $\mathbf{b} \in\left[L^{\infty}(\Omega)\right]^{2}$ is solenoidal in $\Omega$ and such that

$$
\mathbf{b} \cdot \mathbf{n} \geq 0 \quad \text { on } \Gamma_{N} .
$$

Assume also that

$$
0<\kappa_{1}<\frac{\kappa_{2}}{2\|\mathbf{b}\|_{\left[L^{\infty}(\Omega)\right]^{2}}^{2}}, \quad 0<\kappa_{2}<\epsilon \quad \text { and } \quad \kappa_{3}>\frac{1}{2}\|\mathbf{b} \cdot \mathbf{n}\|_{L^{\infty}\left(\Gamma_{D}\right)} .
$$

Then, problem (4) has a unique solution, $\left(\sigma_{0}, u\right) \in \mathbf{H}$.

Proof. It is a consequence of the Lax-Milgram Lemma.

\section{Augmented mixed finite element method}

In what follows, we let $\left\{\mathcal{T}_{h}\right\}_{h>0}$ be a family of shape-regular meshes of $\bar{\Omega}$ made up of triangles; we denote by $h_{T}$ the diameter of an element $T \in \mathcal{T}_{h}$ and define $h:=\max _{T \in \mathcal{T}_{h}} h_{T}$. Given $T \in \mathcal{T}_{h}$ and an integer $l \geq 0$, we denote by $\mathcal{P}_{l}(T)$ the space of polynomials of total degree at most $l$ defined on $T$ and, given an integer $r \geq 0$, we denote by $\mathcal{R} \mathcal{T}_{r}(T)$ the local Raviart-Thomas space of order $r$ (cf. [11]). Then, we define $H_{h}:=\mathcal{R} \mathcal{T}_{r}=\left\{\tau_{h} \in H_{0}\right.$ : $\left.\left.\tau_{h}\right|_{T} \in \mathcal{R} \mathcal{T}_{r}(T), \quad \forall T \in \mathcal{T}_{h}\right\}$, or $H_{h}:=\mathcal{B D}_{\mathcal{M}_{r+1}}=\left\{\tau_{h} \in H_{0}:\left.\tau_{h}\right|_{T} \in\right.$ $\left.\left[\mathcal{P}_{r+1}(T)\right]^{2}, \quad \forall T \in \mathcal{T}_{h}\right\}$ (see [3]). On the other hand, let $m \geq 1$ and define $V_{h}:=\mathcal{L}_{m}=\left\{v_{h} \in \mathcal{C}(\bar{\Omega}):\left.v_{h}\right|_{T} \in \mathcal{P}_{m}(T), \quad \forall T \in \mathcal{T}_{h}\right\}$. Then, the Galerkin scheme associated to problem (4) reads: find $\left(\sigma_{0, h}, u_{h}\right) \in \mathbf{H}_{h}:=H_{h} \times V_{h}$ such that

$$
A_{s}\left(\left(\sigma_{0, h}, u_{h}\right),\left(\tau_{h}, v_{h}\right)\right)=F_{s}\left(\tau_{h}, v_{h}\right), \quad \forall\left(\tau_{h}, v_{h}\right) \in H_{h} \times V_{h} .
$$

Under the hypotheses of Theorem 1, problem (9) has a unique solution $\left(\sigma_{0, h}, u_{h}\right) \in H_{h} \times V_{h}$. Moreover, there exists a constant $C>0$, independent of $h$, such that

$$
\left\|\left(\sigma_{0}-\sigma_{0, h}, u-u_{h}\right)\right\|_{\mathbf{H}} \leq C \inf _{\left(\tau_{h}, v_{h}\right) \in H_{h} \times V_{h}}\left\|\left(\sigma_{0}-\tau_{h}, u-v_{h}\right)\right\|_{\mathbf{H}} .
$$

The corresponding rate of convergence is given in the next theorem.

Theorem 2. Assume $\sigma_{0} \in\left[H^{t}(\Omega)\right]^{d}$, $\operatorname{div}\left(\sigma_{0}\right) \in H^{t}(\Omega)$ and $u \in H^{t+1}(\Omega)$. Then, under the assumptions of Theorem 1 , there exists a positive constant $C_{\text {err }}=\mathcal{O}\left(\|\mathbf{b}\|^{2} / \epsilon^{2}\right)$, independent of $h$, such that

$$
\begin{aligned}
& \left\|\left(\sigma_{0}-\sigma_{0, h}, u-u_{h}\right)\right\|_{\mathbf{H}} \leq \\
& \leq C_{\text {err }} h^{\min \{t, m, r+1\}}\left(\left\|\sigma_{0}\right\|_{\left[H^{t}(\Omega)\right]^{d}}+\left\|\operatorname{div}\left(\sigma_{0}\right)\right\|_{H^{t}(\Omega)}+\|u\|_{H^{t+1}(\Omega)}\right) .
\end{aligned}
$$

Proof. It follows straightforwardly from inequality (10) and the approximation properties of the corresponding finite element subspaces. 


\section{A posteriori error indicator}

Let $\left(\sigma_{0, h}, u_{h}\right) \in \mathbf{H}_{h}$ be the unique solution to problem (9). We denote by $E_{\Gamma_{D}}$ the set of all the edges induced by the mesh $\mathcal{T}_{h}$ that are contained in $\bar{\Gamma}_{D}$. Moreover, for each edge $e \in E_{\Gamma_{D}}$, we denote by $h_{e}$ the length of $e$; we also fix a unit normal vector, $\mathbf{n}_{e}:=\left(n_{1}, n_{2}\right)^{\mathrm{t}}$, and let $\mathbf{t}_{e}:=\left(-n_{2}, n_{1}\right)^{\mathrm{t}}$ be the corresponding fixed unit tangential vector along $e$. where

We consider the global a posteriori error indicator $\theta:=\left(\sum_{T \in \mathcal{T}_{h}} \theta_{T}^{2}\right)^{1 / 2}$,

$$
\begin{aligned}
\theta_{T}^{2} & :=\left\|f+\operatorname{div}\left(\sigma_{z}+\sigma_{0, h}\right)-\mathbf{b} \cdot \nabla u_{h}\right\|_{L^{2}(T)}^{2}+\left\|\nabla u_{h}-\frac{1}{\epsilon}\left(\sigma_{z}+\sigma_{0, h}\right)\right\|_{\left[L^{2}(T)\right]^{2}}^{2} \\
& +\sum_{e \in E_{\Gamma_{D}} \cap \partial T} h_{e}\left(\left\|g-u_{h}\right\|_{L^{2}(e)}^{2}+\left\|\frac{\partial}{\partial \mathbf{t}_{e}}\left(g-u_{h}\right)\right\|_{L^{2}(e)}^{2}\right)
\end{aligned}
$$

We remark that $\theta_{T}$ consists of two terms in interior elements and elements with a side on the Neumann boundary, whereas it contains two additional terms on elements with a side on the Dirichlet boundary.

In the next theorem we establish the reliability and local efficiency of $\theta$.

Theorem 3. Let $\left(\sigma_{0}, u\right) \in \mathbf{H}$ and $\left(\sigma_{0, h}, u_{h}\right) \in \mathbf{H}_{h}$ be the unique solutions to problems (4) and (9), respectively. Then, there exists a positive constant $C_{\mathrm{rel}}$, independent of $h$, such that

$$
\left\|\left(\sigma_{0}-\sigma_{0, h}, u-u_{h}\right)\right\|_{\mathbf{H}} \leq C_{\mathrm{rel}} \theta
$$

Moreover, if $g \in H^{1}\left(\Gamma_{D}\right)$ is a piecewise polynomial on $\Gamma_{D}$, then there exists $C_{\text {eff }}>0$, independent of $h$, such that for all $T \in \mathcal{T}_{h}$ we have

$$
\theta_{T}^{2} \leq C_{\text {eff }}^{-1}\left(\left\|u-u_{h}\right\|_{H^{1}(T)}^{2}+\left\|\sigma_{0}-\sigma_{0, h}\right\|_{H(d i v, T)}^{2}\right)
$$

Proof. See [7]. In the convection-dominated regime, $C_{\text {rel }}=\mathcal{O}\left(\|\mathbf{b}\|^{3} / \epsilon\right)$ and $C_{\text {eff }}=\mathcal{O}\left(\epsilon^{2}\right)$.

\section{$5 \quad$ Numerical experiments}

In this section, we deal with the solution of the boundary value problem

$$
\left\{\begin{aligned}
-\epsilon \Delta u+\mathbf{b} \cdot \nabla u=0 & \text { in } \Omega:=(0,1) \times(0,1), \\
u=0 & \text { on } x=0, \\
u=1 & \text { on } x=1, \\
\epsilon \nabla u \cdot \mathbf{n}=0 & \text { on } y=0, \quad y=1,
\end{aligned}\right.
$$


where $\mathbf{b}=(1,0)^{\mathbf{t}}$ and $\epsilon$ ranges from $10^{-2}$ to $10^{-4}$. The exact solution of problem (15) is (see [4])

$$
u(x, y)=\frac{e^{\frac{x}{\epsilon}}-1}{e^{\frac{1}{\epsilon}}-1}, \quad(x, y) \in \Omega .
$$

We remark that $u$ has an exponential boundary layer around the line $x=1$.

We present numerical results for the finite element pairs $\left(H_{h}, V_{h}\right)$ given by $\left(\mathcal{R} \mathcal{T}_{0}, \mathcal{L}_{1}\right),\left(\mathcal{R} \mathcal{T}_{1}, \mathcal{L}_{2}\right),\left(\mathcal{B D} \mathcal{M}_{1}, \mathcal{L}_{1}\right)$ and $\left(\mathcal{B D} \mathcal{M}_{2}, \mathcal{L}_{2}\right)$ in $\mathbb{R}^{2}$. The numerical experiments were performed using the finite element toolbox FEniCS [8]. We compare the performance of the finite element method based on uniform refinement with the adaptive method based on the a posteriori error indicator $\theta$. For the selection of elements to be refined, we rely on the maximum strategy with a threshold $\gamma=0.4$. We choose $\kappa_{1}=\frac{\epsilon}{8}, \kappa_{2}=\frac{\epsilon}{2}$ and $\kappa_{3}=1$. We remark that these values satisfy conditions (8). Finally, we define the total error

$$
e_{h}\left(\sigma_{0}, u\right):=\left(\left\|\sigma_{0}-\sigma_{0, h}\right\|_{H(\operatorname{div} ; \Omega)}+\left\|u-u_{h}\right\|_{H^{1}(\Omega)}\right)^{1 / 2} .
$$

In Figure 1, we report the total error and estimator for the uniform and adaptive refinements. From these graphs, we conclude that the adaptive algorithm is more competitive than the uniform procedure. Figure 2 shows the efficiency indices for the different finite elements and the different values of $\epsilon$ considered here. We note that their values stay in the same range for all values of $\epsilon$ and all elements considered. In Figure 3 we show the initial, an intermediary and final meshes for $\epsilon=10^{-4}$. We observe that the adaptive algorithm is able to locate the boundary layer of the solution.

Finally, Figure 4 shows the final concentration and flux obtained in that case with the adaptive procedure.

\section{Acknowledgements}

The research of the first author was partially supported by MICINN grant MTM2016-76497-R. The research of the second author was supported by the Basque Government through the BERC 2014-2017 programme and by the Spanish Ministry of Economy and Competitivity through the BCAM Severo Ochoa excellence accreditation SEV-2013-0323.

\section{References}

1. T.P. Barrios, J.M. Cascón And M. GonzÁlez, A posteriori error analysis of an augmented mixed finite element method for Darcy flow, Comput. Methods Appl. Mech. Engrg. 283 (2015) 909-922.

2. ___ Augmented mixed finite element method for the Oseen problem: A priori and a posteriori error analyses, Comput. Methods Appl. Mech. Engrg. 313 (2017) 216-238. 

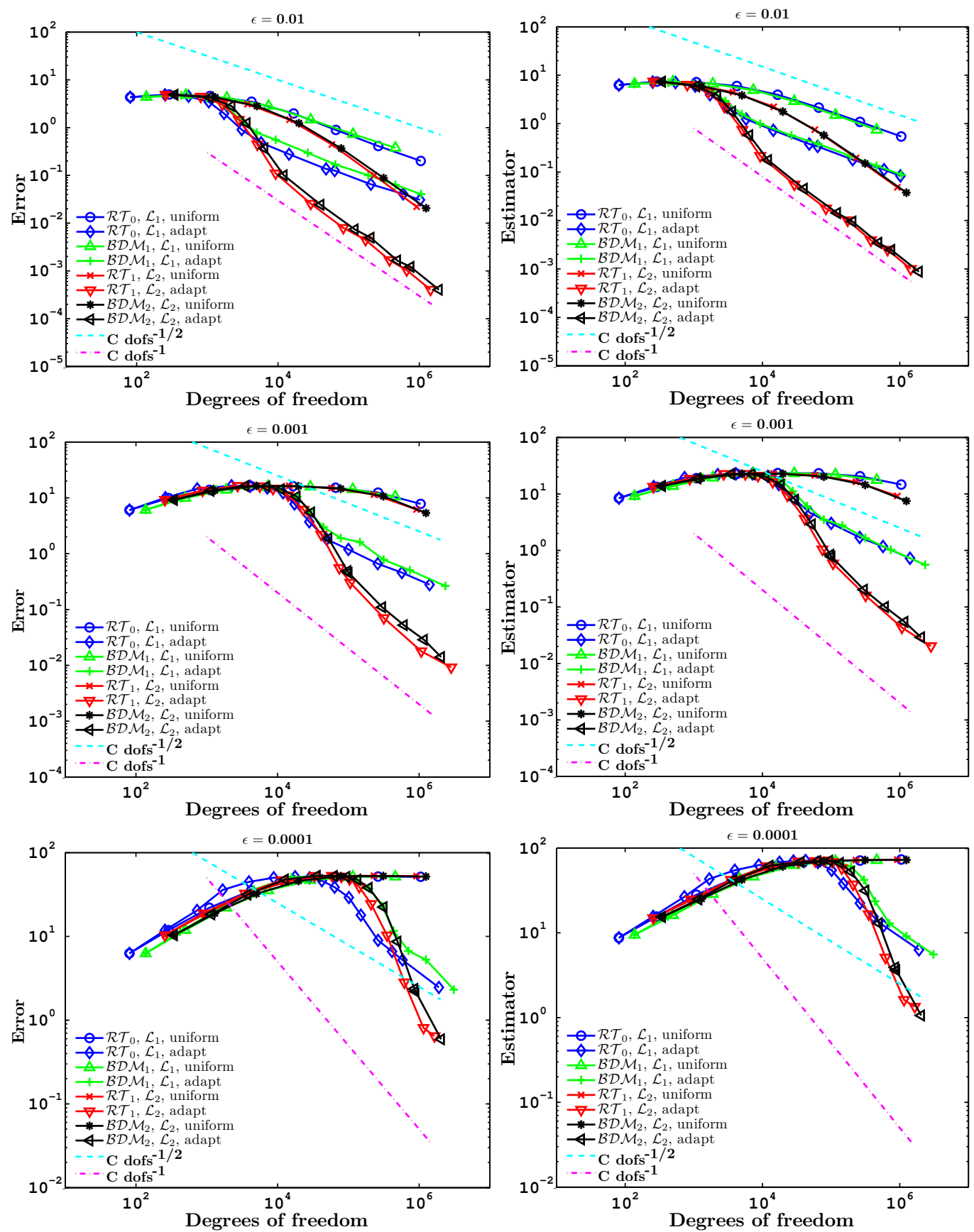

Fig. 1. Decay of error (left) and estimator (right) vs. number of degrees of freedom for three values of $\epsilon$ and different elements. 

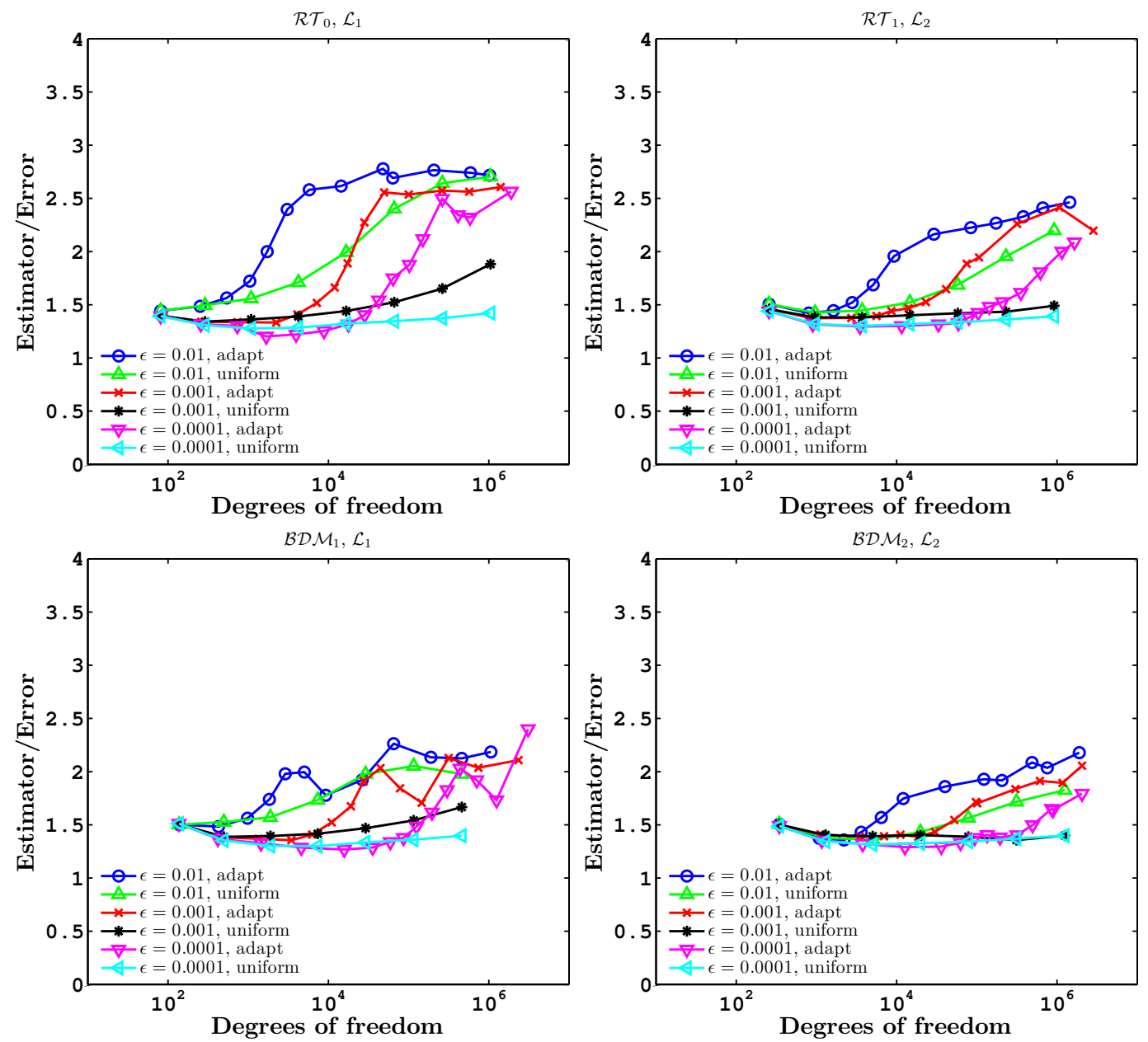

Fig. 2. Efficiency indices for different finite elements.

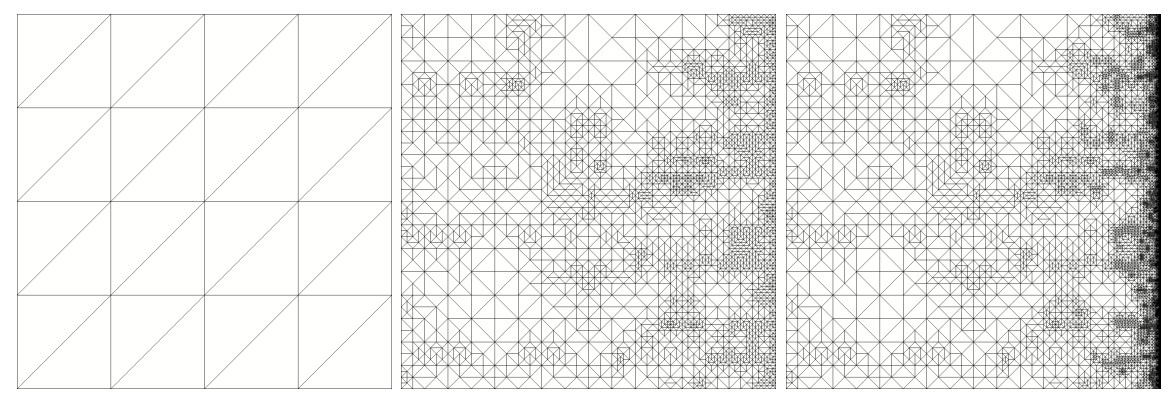

Fig. 3. Initial, intermediary (after 5) and final (after 15 iterations) mesh for $\epsilon=$ 0.0001 when using $\left(\mathcal{R} \mathcal{T}_{0}, \mathcal{L}_{1}\right)$. 

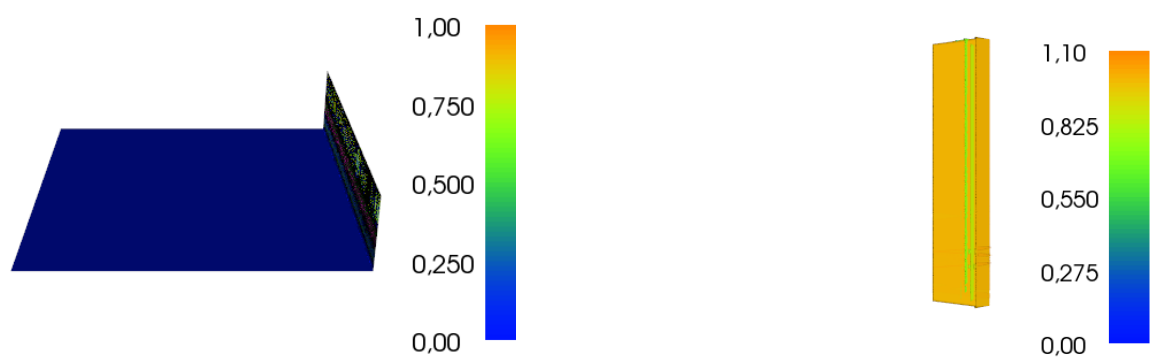

Fig. 4. Final concentration (left) and flux (right) for $\epsilon=0.0001$ when using $\left(\mathcal{R} \mathcal{T}_{0}, \mathcal{L}_{1}\right)$.

3. F. Brezzi And M. Fortin, Mixed and Hybrid Finite Element Methods, Springer-Verlag, 1991.

4. M. Farhloul and A.S. Mounim, A mixed-hybrid finite element method for convection-diffusion problems, Appl. Math. Comput. 171 (2005) 1037-1047.

5. M. GonzÁLEz, Stabilized dual-mixed method for the problem of linear elasticity with mixed boundary conditions, Applied Mathematics Letters 30 (2014) 1-5.

6. M. GonzÁlez, S. Korotov And J. Jansson, A posteriori error analysis of a stabilized mixed FEM for convection-diffusion problems, Discrete Contin. Dyn. Syst. (2015), Dynamical systems, differential equations and applications. 10th AIMS Conference. Suppl., 525-532.

7. M. GonzÁlez And M. Strugaru, Stabilization and a posteriori error analysis of a mixed FEM for convection-diffusion problems with mixed boundary conditions, in preparation.

8. A. Logg, K.-A. Mardal and G.N. Wells Ed., Automated Solution of Differential Equations by the Finite Element Method. The FEniCS Book, Springer, 2012 .

9. A. Masud And T.J.R. Hughes, A stabilized mixed finite element method for Darcy flow, Comput. Methods Appl. Mech. Engrg. 191 (2002) 4341-4370.

10. A. PlazA AND G.F. CAREY, Local refinement of simplicial grids based on the skeleton, Appl. Numer. Math. 32 (2000) 195-218.

11. J.E. Roberts And J.-M. Thomas, Mixed and Hybrid Methods, in Handbook of Numerical Analysis, edited by P.G. Ciarlet and J.L. Lions, vol. II, Finite Element Methods (Part 1). North-Holland, Amsterdam (1991).

12. J.-M. Thomas, Mixed finite elements methods for convection-diffusion problems, Numerical Approximation of Partial Differential Equations, Elsevier (1987) 241-250. 